\title{
The Effects of a Learning Analytics Empowered Technology on Students' Arithmetic skill Development
}

\author{
Inge Molenaar \\ Radboud University \\ Montessorilaan 3 \\ Nijmegen \\ +3124-3611942 \\ i.molenaar@pwo.ru.nl
}

\author{
Carolien A. N. Knoop-van Campen \\ Radboud University \\ Montessorilaan 3 \\ Nijmegen \\ +3124-3615511 \\ c.knoop-vancampen@pwo.ru.nl
}

\author{
Fred Hasselman \\ Radboud University \\ Montessorilaan 3 \\ Nijmegen \\ +3124-3611942 \\ f.hasselman@pwo.ru.nl
}

\begin{abstract}
Learning analytics empowered educational technologies (LA-ET) in primary classrooms allow for blended learning scenarios with teacher-lead instructions, class-paced and individually-paced practice. This quasi-experimental study investigates the effects of a LA-ET on the development of students' arithmetic skills over one schoolyear. Children learning in a traditional paper \& pencil condition were compared to learners using a LA-ET on tablet computers in grade 4 . The educational technology combined teacher dashboards (extracted analytics) and class and individually paced assignments (embedded analytics). The results indicated that children in the LA-ET condition made significantly more progress on arithmetic skills in one schoolyear compared to children in the paper \& pencil condition.
\end{abstract}

\section{Categories and subject descriptors \\ - Applied computing E-learning}

\section{General Terms}

Algorithms, Experimentation, Human Factors, Standardization, Theory, Verification.

\section{Keywords}

Educational technologies, primary education, arithmetic's, ability levels

\section{INTRODUCTION}

Even though the recent influx of tablets in primary education is based on the vision that educational technology empowered with learning analytics (LA-ET) will revolutionize education, empirical results supporting this claim are scarce [1]. Specifically, these advances are expected to enhance the effectiveness of education [2, 3]. The present study investigates the effects of an LA_ET on the development of children's arithmetic skills compared to a traditional paper \& pencil learning environment in grade 4 of primary education. Most research on effectiveness of educational technologies entails so-called individually paced technologies: i.e. these technologies adapt pace and difficulty of assignments to students' skills. Computer Assisted Instruction (CAI) and Intelligent Tutoring Systems (ITS) are both examples of

Permission to make digital or hard copies of part or all of this work for personal or classroom use is granted without fee provided that copies are not made or distributed for profit or commercial advantage and that copies bear this notice and the full citation on the first page. Copyrights for third-party components of this work must be honored. For all other uses, contact the Owner/Author.

Copyright is held by the owner/author(s).

LAK '17, March 13-17, 2017, Vancouver, BC, Canada

ACM 978-1-4503-4870-6/17/03.

http://dx.doi.org/10.1145/3027385.3029488 individually paced technologies [3]. A meta-analysis evidenced that comprehensive blended learning scenarios, in which CAI or ITS are combined with classroom activities, elicit larger learning gains, compared to using educational technologies in isolation [3]. Yet, both CAI and ITS are not often used in blended scenarios combining teacher-lead instructions, class-paced and individuallypaced practice. This can be explained by the fact that CAI and ITS are both individually paced, which entails that students learn and advance at different speed. For teachers, it is difficult to connect individually paced practice with classroom-wide activities that are meaningful for all students. Extracted analytics in the form of dashboards can support teachers to view class wide as well as students' individual progress. This can function as a bridge to combine class-paced activities (instruction and practice) and individually paced practice.

\section{THE PRESENT STUDY}

Hence teaching with LA-ET can support blended educational scenarios in primary education. These scenarios combine teacherlead instructions, class-paced and individually-paced practice. In this study, the learning analytics empowered educational technology provided teachers with dashboards that gave an overview of class and student progress. Moreover, individual practice was adjusted to the students' current performance level. This quasi-experimental study investigates the effect of using this LA-ET on the development of students' arithmetic skills in grade 4 over one schoolyear. Children learning in a traditional paper \& pencil condition were compared to children using a LA-ET on tablet computers. We expect that on average, skill development will be larger for the students who work with LA-ET compared to the paper \& pencil condition. This hypothesis is based on positive effects of earlier CAI and ITS studies [3] and research indicating the benefits of applying CAI in blended learning scenarios [3]. Additionally, dashboards (extracted analytics) are expected to support teachers to connect teacher-lead instructions, class-paced and individually-paced practice, which can also enhance students' arithmetic's skill development.

\section{METHOD}

\subsection{Participants}

In this study, 41 schools participated, with 1579 children in grade 4. Informed consent was obtained from the schools before students were allowed to participate. Students data was stored anonymously.

\subsection{Learning analytics empowered technology}

The LA-ET used in this study is called 'Snappet'. This technology is used for both arithmetic and language education across schools in the Netherlands. The practice activities are comparable between conditions as the arithmetic assignments in the LA-ET are 
comparable to those used in the paper \& pencil workbooks. The technology operates on tablet computers and features both embedded and extracted learning analytics. Class and individually paced assignments are available and children receive immediate feedback after finishing each assignment. Individually paced assignments are adjusted automatically to students' current performance level. Teachers have access to 3 different types of dashboards with which they can monitor class and student progress.

\subsection{MEASURMENTS}

\subsubsection{Arithmetic skill development and ability levels}

The Dutch national standardized arithmetic assessment, CITO Rekenen-Wiskunde 2.0 [Arithmetic-Mathematics] was used to measure children's arithmetic. Children's raw scores on these assessments were thus converted into national standardized normed ability scores that showed the degree to which the student masters arithmetic skills.

\subsection{Procedure}

The data collection took place over one full schoolyear from June 2015 to June 2016. The standardized test scores (CITO) are from June 2015 (Time 1) and June 2016 (Time 2).

\subsection{Data-analysis}

To examine the effect of LA-ET on students' arithmetic skill development over one schoolyear, a random effects model was with Time (Time 1 / Time 2) as within-subject-factor, Condition (LAET / Control) as between-subject-factor, and Subject and School as random effects.

\section{RESULTS}

Analysis of arithmetic skills showed a significant effect of Condition and an interaction effect of Time X Condition. Disregarding Condition there was a significant increase in scores over time, $t(1427.7)=34.33, p<.001$. The students in both conditions made significant progress during the year. Disregarding Time, no significant difference was found between the LA-ET and the Control condition, $t(39.3)=-1.83, \mathrm{p}=0.13$. Students in both conditions scored equally high on arithmetic skills. The interaction effect between Time and Condition was significant, $t(1427.6)=-$ $8.15, \mathrm{p}<0.001$. Students in the LA-ET condition made more progress on arithmetic skills than students in the control condition (see Table 1 and Figure 1).

Table 1. Arithmetic Skills per Condition

\begin{tabular}{cccccccc}
\hline & \multicolumn{3}{c}{ Time 1 } & \multicolumn{5}{c}{ Time 2 } & Progress \\
\hline & $\mathrm{N}$ & $\mathrm{M}$ & $\mathrm{SD}$ & $\mathrm{N}$ & $\mathrm{M}$ & $\mathrm{SD}$ & \\
\hline LA-ET & 742 & 80.39 & 14.68 & 778 & 94.64 & 12.54 & 14.25 \\
Control & 730 & 81.66 & 13.41 & 727 & 92.20 & 11.76 & 10.54 \\
\hline
\end{tabular}

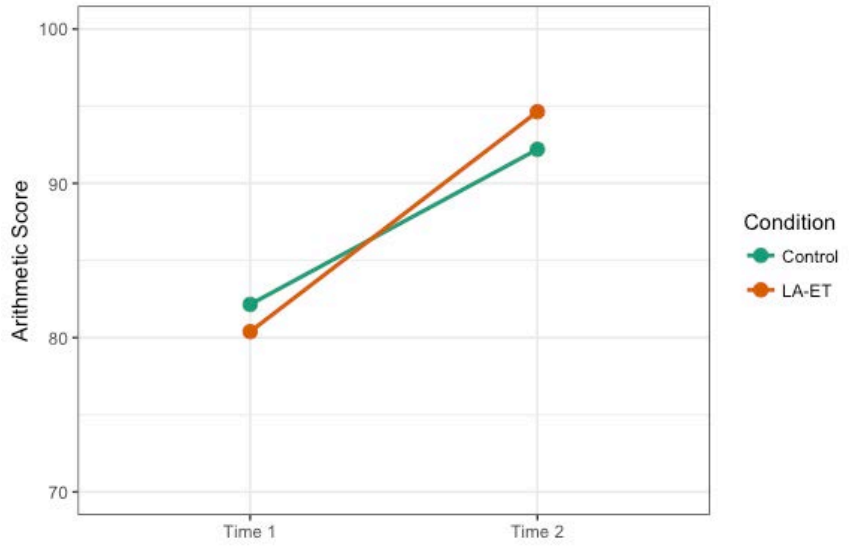

Figure 1 Arithmetic Skills per Condition over Time

\section{DISCUSSION}

In this study, we examined the effect of an LA-ED with both embedded and extracted analytics on the development of arithmetic skills over one schoolyear. The results indicated that children in the LA-ET condition made significantly more progress on arithmetic skills in one schoolyear compared to children in the paper \& pencil condition. This supports the notion that educational technologies that translate data into actionable information for teachers indeed support student learning. Moreover, in this study we learned that educational technologies combining extracted and embedded learning analytics did indeed support blended education scenarios including teacher-lead instructions, class-paced and individuallypaced practice. However, many variations in blended scenario's were noticed, which stresses the need for advanced efforts to balance embedded analytics and extracted analytics in such a way that system strengths and human strengths together optimally support learning in learning analytics empowered technologies in primary education.

\section{ACKNOWLEDGMENTS}

We give thanks to Kennisnet for financial support for this study.

\section{REFERENCES}

[1] A. Papamitsou, Z. Economides, A. Anastasios. 2014. Learning Analytics and Educational Data Mining in Practice: A Systematic Literature Review of Empirical Evidence. Journal of Educational Technology \& Society, 17(4), 49-64.

[2] D. Tempelaar, B. Rienties, B. Giesbers. 2015. In search for the most informative data for feedback generation: Learning Analytics in a data-rich context. Computers in Human Behavior, 47, 157-167.

[3] K. van Lehn. 2006. The behavior of tutoring systems. International Journal of Artificial Intelligence in Education. 16 (3), 227-265.

[4] G. Siemens. 2013. Learning analytics: The emergence of a discipline. American Behavioral Scientist, 57, 13801400 . 